\title{
Effects of atrioventricular asynchrony on platelet activation: implication of thromboembolism in paced patients
}

Chu-Pak Lau, Hung-Fat Tse, Gregory Cheng

\begin{abstract}
Objective-To investigate the platelet activation in different modes of pacing in patients implanted with dual chamber rate adaptive pacemaker (DDDR) for bradyarrhythmias, and to explore the possible underlying mechanism of the higher thromboembolic incidence in single chamber ventricular rate adaptive (VVIR) pacing.
\end{abstract}

Design-Platelet activation was determined in chronically paced patients during three different pacing modes (VVIR, DDD, and DDDR) in a randomised crossover fashion.

Setting-Pacemaker clinic at a university teaching hospital.

Patients-15 patients with complete heart block, mean (SD) age 63 (10) years, and 12 patients with sick sinus syndrome, mean age 68 (9) years, implanted with DDDR pacemakers.

Main outcome measures-Platelet activation was assessed by measuring the plasma concentrations of platelet factor 4 (PF4) and $\beta$ thromboglobulin using an enzyme linked immunosorbent assay (ELISA). Mean log plasma PF4 and $\beta$ thromboglobulin values were compared in paced patients during different pacing modes and with control.

Results-Compared with controls, patients paced in DDDR, DDD, and VVIR modes had higher mean log plasma concentrations of PF4 (0.90 (0.32), $0.92(0.29)$, and $1.12(0.33) v 0.61(0.29) \log \mathrm{IU} / \mathrm{ml}$, all $\mathrm{p}<0.05$, respectively) and $\beta$ thromboglobulin (1.55 (0.20), $1.59(0.16)$, and $1.71(0.18) \quad v \quad 1.40(0.12) \quad \log$ IU $/ \mathrm{ml}$, all $p<0.05$, respectively). In paced patients, VVIR pacing was associated with higher plasma concentrations of PF4 and $\beta$ thromboglobulin than either DDDR or DDD pacing (all $p<0.05$ ). There was no significant difference in plasma PF4 and $\beta$ thromboglobulin between patients with complete heart block and sick sinus syndrome in the corresponding pacing mode. Holter monitoring showed no difference in mean pacing rate and occurrence of cardiac arrhythmias to account for the increased platelet activation during VVIR pacing. There was no relation between the percentage of ventricular pacing on Holter during DDDR, DDD, and VVIR modes and the log mean plasma concentrations of PF4 $(r=0.002,0.001$, and 0.001 , respectively, all $p>0.05$ ) and $\beta$ thromboglobulin $(r=0.007,0.01$, and 0.001 , respectively, all $\mathrm{p}>0.05$ ).

Conclusions-Single chamber ventricular pacing was associated with enhanced spontaneous systemic platelet activation compared with physiological dual chamber pacing. This was related to the loss of atrioventricular synchrony rather than to the underlying cause of bradycardia, lack of rate response, or coexisting arrhythmia. This abnormality may be associated with increased thromboembolism and was correctible by an appropriate pacing mode prescription and possibly antiplatelet treatment.

(Heart 1997;78:358-363)

Keywords: pacing; thromboembolism; platelet activation

Single chamber ventricular (VVI) pacing is effective in preventing some bradycardia related symptoms in patients with bradyarrhythmias. ${ }^{1-3}$ However, previous studies have suggested that the incidence of thromboembolism is higher with a ventricular than with an atrial pacing mode in patients with bradyarrhythmias. ${ }^{4-10}$ This may be related to the greater frequency of atrial arrhythmias or abnormal atrial mechanical and transport function during ventricular pacing. Previous studies $^{11} 12$ have shown that patients suffering from stroke or transient ischaemic attacks have increased platelet activation, and antiplatelet agents have been effective in preventing further cerebrovascular events in these patients. ${ }^{13}$ It is uncertain whether a similar mechanism operates in patients with bradycardia fitted with a pacemaker. Dual chamber rate adaptive pacemaker (DDDR) pacemakers allow noninvasive programming of pacing modes to examine the electrical and physiological effects of atrioventricular synchrony and rate adaptation. This study was designed to compare the platelet activation in different modes of pacing in patients implanted with DDDR pacemakers for bradyarrhythmias and to explore the possible underlying mechanism for the higher thromboembolic incidence in VVI pacing.

Methods

PATIENTS

The study group consisted of 15 patients with complete heart block, mean (SD) age 63 (10) 


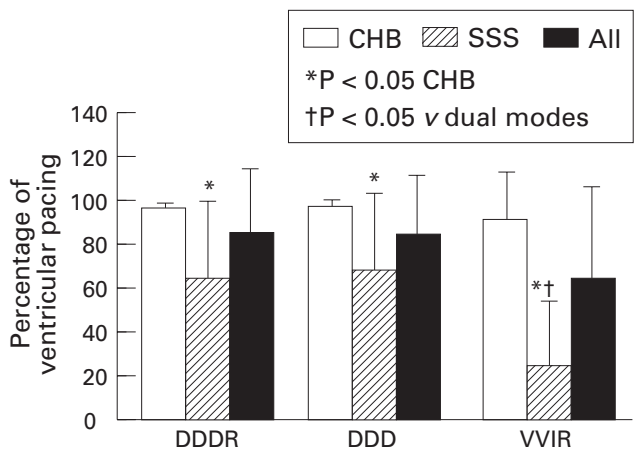

Figure 1 Percentage of ventricular pacing in different pacing modes in patients with sick sinus syndrome (SSS; $n=12)$ and complete heart block $(C H B ; n=15)$.

years (ten women, five men), and 12 patients with sick sinus syndrome, mean age 68 (9) years (seven women, five men), implanted with either activity sensing or minute ventilation sensing DDDR pacemakers. None of them had documented atrial tachyarrhythmias on resting electrocardiogram (ECG) or 24 hour Holter monitoring before implantation. All had normal left ventricular function on radionuclide ventriculography (mean ejection fraction $54.4(9.1) \%)$. Five patients had hypertension. None had histories of abnormalities such as cerebrovascular disease, renal failure, and diabetic mellitus known to be associated with platelet activation. ${ }^{14}$ The control group consisted of 16 healthy subjects, mean age 66 (11) years (eight women, eight men), all normal on physical examination and on resting electrocardiographic and echocardiographic study. There was no significant difference in age and sex distribution between patients and controls. Written informed consent was obtained from all participants, and the study was approved by the local ethics committee.

STUDY PROTOCOL AND PACEMAKER

PROGRAMMING

After checking pacemaker function, resting 12 lead ECG and Holter monitoring was done at baseline. The pacemakers were then programmed into three different pacing modes in a randomised double blind protocol, each for an eight week study period as below:

DDDR mode - Both atrioventricular synchrony and sensor driven rate adaptation.

DDD mode-Atrioventricular universal pacing, allowing atrioventricular synchrony and sinus determined rate response.

VVIR mode - Single chamber ventricular rate adaptive mode (that is, no atrioventricular synchrony) but sensor driven rate adaptive response.
After eight weeks of pacing in each mode, 24 hour Holter monitoring was performed in each patient, followed by blood sampling for platelet activation (see below).

HOLTER RECORDING

Bipolar electrocardiographic chest leads were fixed to the chest at the V1 and V6 positions, and all electrograms were recorded for 24 hours using an Oxford Medilog recorder (Oxford Medical Instruments, Oxford, UK). The electrocardiographic pattern was sampled for one minute over the first and 30th minute of each hour and printed at a paper speed of 25 $\mathrm{mm} / \mathrm{s}$. Right ventricular capture was defined by the presence of a pacing spike followed by a QRS complex with a width $\geqslant 12 \mathrm{~ms}$. The percent of QRS complexes with ventricular capture during each observation minute was averaged over the 24 hour period. The incidence and frequency of cardiac arrhythmias were also analysed using automatic analysis and full disclosure review.

\section{PLATELET ACTIVATION STUDY}

Platelet activation was assessed by measuring plasma concentrations of both platelet factor 4 (PF4) and $\beta$ thromboglobulin, which are platelet specific proteins secreted from the $\alpha$ granules during the release reaction. ${ }^{14}{ }^{15} \mathrm{PF} 4$ binds to the endothelial surface heparin-like molecules and hence has a short half life, whereas $\beta$ thromboglobulin is not bound by vascular endothelium and is cleared from the circulation by renal excretion. Both proteins can be accurately measured by enzyme immunoassay providing that specific precautions (see below) have been taken to prevent in vitro activation during phlebotomy and plasma processing. ${ }^{15}$ We monitored this by measuring PF4 and $\beta$ thromboglobulin concentrations. A high concentration of $\beta$ thromboglobulin in the presence of a normal or only slightly raised PF4 strongly suggests in vivo release, while a comparable increase in both proteins suggests in vitro release. The ratio of $\beta$ thromboglobulin to PF4 was used to exclude in vitro activation, a significant increase in the ratio (above 3:1) suggesting in vivo activation. ${ }^{16}{ }^{17}$ Blood results with evidence of in vitro platelet activation $(\beta$ thromboglobulin to PF4 ratio less than 3:1) were not included for analysis.

Patients and controls were instructed to abstain from any drug treatment or alcohol for 10 days before blood sampling. All blood samples were taken in the morning for convenience of processing. Precautions were taken to avoid ex vivo activation of platelets. Thus blood samples were drawn by venepuncture using a 21

Table 1 Percentage of ventricular pacing and mean heart rate on $24 \mathrm{~h}$ Holter monitoring

\begin{tabular}{|c|c|c|c|c|c|c|}
\hline & \multicolumn{3}{|c|}{ Sick sinus syndrome $(n=12)$} & \multicolumn{3}{|c|}{ Complete heart block $(n=15)$} \\
\hline & $D D D R$ & $D D D$ & $V V I R$ & $D D D R$ & $D D D$ & $V V I R$ \\
\hline Pacing (\%) & $70.3(36.7)$ & $74.9(36.3)$ & $48.1(39.8)^{\star}$ & $99.7(1.01) \dagger$ & $92.9(24.9) \dagger$ & $92.9(21.3) \dagger$ \\
\hline Mean heart rate (beats/min) & $72.3(13.2)$ & $70.4(10.2)$ & $65.3(9.8)$ & $72.3(12.5)$ & $70.4(9.8)$ & $68.5(12.3)$ \\
\hline
\end{tabular}

Values are mean (SD).

${ }^{\star} \mathrm{p}<0.05 v \mathrm{DDD} / \mathrm{DDDR}$ mode in patients with sick sinus syndrome.

$\mathrm{tp}<0.05 v$ corresponding pacing modes in sick sinus syndrome. 
Table 2 Comparison of platelet activation during different modes of pacing (DDDR, DDD, and VVIR) with controls

\begin{tabular}{|c|c|c|c|c|}
\hline & $D D D R(n=27)$ & $D D D(n=27)$ & $\operatorname{VVIR}(n=27)$ & Control $(n=16)$ \\
\hline PF4 (IU/ml) 95\% CI & 7.30 to 13.17 & 7.76 to 12.29 & 17.24 (13.68) 11.83 to 22.65 & $4.96(3.14) \quad 3.28$ to 6.63 \\
\hline $\log [\mathrm{PF} 4](\mathrm{IU} / \mathrm{ml}) 95 \% \mathrm{CI}$ & $0.90(0.32)^{\star} 0.78$ to 1.03 & $0.92(0.29) \dagger 0.81$ to 1.04 & $1.12(0.33) \dagger 0.99$ to 1.25 & $0.61(0.29) \quad 0.45$ to 0.77 \\
\hline BTG (IU/ml) $95 \%$ CI & $39.48(19.82) 31.63$ to 47.32 & 41.11 (15.72) 34.89 to 47.33 & $55.58(24.69) 45.81$ to 65.35 & 25.77 (6.61) 22.25 to 29.29 \\
\hline $\log [\mathrm{BTG}](\mathrm{IU} / \mathrm{ml}) 95 \% \mathrm{CI}$ & $1.54(0.21)^{\star} \quad 1.47$ to 1.63 & $1.59(0.16) \dagger 1.52$ to 1.65 & $1.71(0.18) \dagger 1.64$ to 1.78 & $1.40(0.12) \quad 1.33$ to 1.46 \\
\hline
\end{tabular}

Values are mean (SD) and $95 \%$ confidence intervals (CI)

BTG, $\beta$ thromboglobulin; PF4, platelet factor 4 .

${ }^{\star} \mathrm{p}<0.01,+\mathrm{p}<0.001 v$ control.

gauge indwelling butterfly needle without using a tourniquet. The first $2 \mathrm{ml}$ of blood were discarded, and then 4-5 $\mathrm{ml}$ of blood were collected into Diatube $\mathrm{H}$ tubes (Diagnostic Stago, France). Tubes were immediately placed in ice water baths for at least 15 minutes. They were then centrifuged at $2500 \mathrm{~g}$ for 30 minutes at $4^{\circ} \mathrm{C}$. After centrifugation, approximately one third of the plasma supernatant was collected by placing the pipette tip in the middle region of the supernatant. This was to avoid aspirating light platelets that might have risen to the surface of the plasma or platelets resting on top surface of the cell layer. The platelet-poor plasma was stored at $-20^{\circ} \mathrm{C}$. PF4 and $\beta$ thromboglobulin assays were carried out within one month of collection of the sample. PF4 assay-PF4 determination was performed by enzyme immunoassay methods using a commercially available kit (Asserachrom PF4 and $\beta$ thromboglobulin, Diagnostica Stago, France) according to manufacturer's instruction. Platelet-poor plasma was incubated in a plastic well coated with specific rabbit antihuman PF4 for one hour at room temperature (antigen immobilisation). After washing, rabbit anti-PF4 antibody coupled with peroxidase was added to the plastic well to form a $\mathrm{PF} 4 /$ anti-PF4-peroxidase immunoconjugate. The bound peroxidase activity was measured by hydrolysis of the colour substrate orthophenylenediamine in the presence of hydrogen peroxide. The reactions were stopped after three minutes and the intensity of the colour produced was determined by measuring absorbance at $492 \mathrm{~nm}$. The amount of PF4 in the platelet-poor plasma was then determined from an absorbance-PF4 calibration curve.

$\beta$ Thromboglobulin assay $-\beta$ Thromboglobulin was measured with the same commercially available kit used for PF4 (Asserachrom PF4 and $\beta$ thromboglobulin, Diagnostica Stago), as described above. Specific rabbit anti-human $\beta$ thromboglobulin antibodies and anti- $\beta$ thromboglobulin-peroxidase conjugates were used. $\beta$ Thromboglobulin concentrations were determined from an absorbency- $\beta$ thromboglobulin calibration curve.

Both PF4 and $\beta$ thromboglobulin assays were run with the same positive controls each time for calibration and the results were highly reproducible $(>95 \%)$. Some patient samples were run in duplicates and again the results were comparable.

STATISTICAL METHODS

Continuous data were expressed as mean (SD). The mean heart rate and percentage of ventricular pacing in each group were compared with two way analysis of variance. As plasma concentrations of $\beta$ thromboglobulin and PF4 are logarithmically distributed, log transformed data were used for analysis. ${ }^{17} \mathrm{~A}$ Mann-Whitney test was performed to compare Holter data between groups for each pacing mode, and to compare log mean plasma concentrations of PF 4 and $\beta$ thromboglobulin between controls and paced patients during each of the three different pacing modes. Multiple comparisons between the mean log plasma concentrations of PF4 and $\beta$ thromboglobulin in the three different pacing modes were performed by repeated measures one way analysis of variance (Bonferroni) analysis. Correlation between variables was by linear regression. A p value $<0.05$ was considered significant.

\section{Results}

HOLTER MONITORING

Results are shown in table 1. Two patients $(7.4 \%)$ with sick sinus syndrome developed episodes of paroxysmal atrial fibrillation, one during DDDR mode (for two minutes) and another during VVIR mode (for 15.5 minutes). Patients with complete heart block had a higher percentage of ventricular pacing on

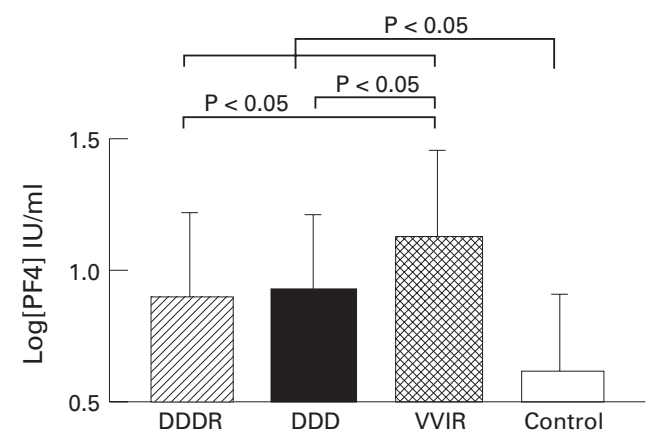

Figure 2 Logarithmically transformed plasma level of platelet factor 4 (PF4) in different pacing modes (DDDR, $D D D, V V I R)$ and controls.

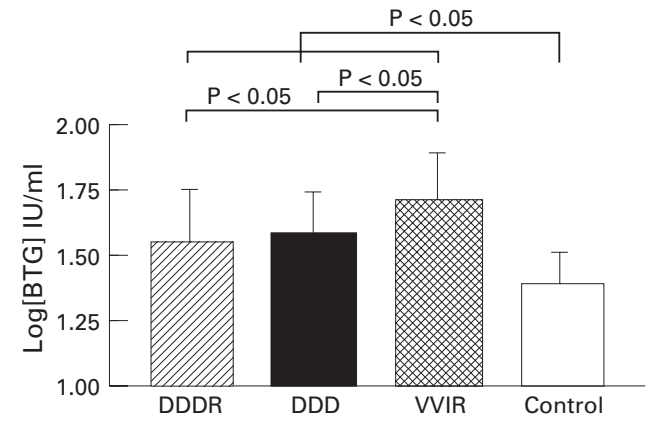

Figure 3 Logarithmically transformed plasma level of $\beta$ thromboglobulin (BTG) in different pacing modes (DDDR, DDD, VVIR) and controls. 
Table 3 Comparison of plaletet activation between patients with complete heart block (CHB) and sick sinus syndrome (SSS) during different modes of pacing

\begin{tabular}{|c|c|c|c|c|}
\hline & Pacing mode & $C H B(n=15)$ & $\operatorname{SSS}(n=12)$ & $p$ value \\
\hline \multirow[t]{3}{*}{$\overline{\log [\mathrm{PF} 4] \mathrm{IU} / \mathrm{ml}(\mathrm{PF} 4 \mathrm{IU} / \mathrm{ml})}$} & DDDR & $0.88(0.36) 10.37(8.10)$ & $0.93(0.26) 10.08(6.81)$ & NS \\
\hline & DDD & $0.86(0.33) 9.28(6.36)$ & $0.99(0.22) 10.95(4.91)$ & NS \\
\hline & VVIR & $1.14(0.28) 16.77(10.42)$ & $1.03(0.36) 14.63(14.75)$ & NS \\
\hline \multirow[t]{3}{*}{$\log$ [BTG] IU/ml (BTG IU/ml) } & DDDR & $1.54(0.16) 40.55(23.40)$ & $1.55(0.16) 38.14(15.11)$ & NS \\
\hline & DDD & $1.59(0.14) 41.19(18.30)$ & $1.59(0.14) 40.27(12.92)$ & NS \\
\hline & VVIR & $1.62(0.19) 60.39(22.11)$ & $1.62(0.19) 45.47(24.52)$ & NS \\
\hline
\end{tabular}

Values are mean (SD).

BTG, $\beta$ thromboglobulin; PF4, platelet factor 4

Holter than patients with sick sinus syndrome in the corresponding modes $(p<0.05) . \mathrm{Pa}-$ tients with sick sinus syndrome had a lower percentage of ventricular pacing during VVIR modes than during DDD and DDDR modes (fig $1 ; \mathrm{p}<0.05$ ). There was no significant difference in mean 24 hour heart rate during different pacing modes in patients with complete heart block and sick sinus syndrome.

PLATELET ACTIVATION STUDY

Comparison with controls

Results are shown in table 2 and in figs 2 and 3. Plasma concentrations of PF 4 and $\beta$ thromboglobulin in paced patients during DDDR, DDD, and VVIR modes were higher than in controls, and the rise in $\beta$ thromboglobulin was greater than that of PF4. The log mean plasma concentrations of PF4 and $\beta$ thromboglobulin were significantly raised during pacing compared with controls.

Comparison between different modes of pacing With the paced patients as their own controls, we compared the plasma concentrations of PF4 and $\beta$ thromboglobulin during VVIR, DDD, and DDDR pacing. VVIR pacing was associated with higher log mean plasma PF4 and $\beta$ thromboglobulin than DDDR and DDD pacing (figs 2 and $3 ; \mathrm{p}<0.05$ ). There was no significant difference in log mean plasma PF4 and $\beta$ thromboglobulin between DDDR and DDD pacing.

Effect of underlying causes of bradyarrhythmia and percentage of ventricular pacing

There was no significant difference in plasma PF4 and $\beta$ thromboglobulin values between patients with sick sinus syndrome and patients with complete heart block in the corresponding pacing mode (table 3 ). There was no relation between the percentage of ventricular pacing on Holter during DDDR, DDD, and VVIR modes and the log mean plasma concentrations of PF4 ( $r=0.002,0.001$, and 0.001, respectively; all $\mathrm{p}>0.05)$ and $\beta$ thromboglobulin $(r=0.007,0.01$, and 0.001 , respectively; all $\mathrm{p}>0.05)$.

\section{Discussion}

THROMBOEMBOLISM IN PACED PATIENTS

Both ventricular pacing and dual chamber pacing are effective in preventing some bradycardia related symptoms in patients with bradyarrhythmias. $^{1-4}$ However, pooled data from several retrospective studies have shown that atrial pacing is associated with significantly lower incidence of thromboembolism $(1.6 \% v$
13\%). ${ }^{4518}$ Furthermore, atrial fibrillation occurs much more often with ventricular than with atrial pacing. ${ }^{578}$ Although the development of atrial fibrillation is one of the risk factors for increased thromboembolism in these patients, the ventricular pacing mode remained an independent predictor for stroke after adjusting for the incidence of atrial fibrillation. ${ }^{9}$ A recent prospective study also showed a trend toward more thromboembolic events in ventricular pacing than in atrial pacing, even in paced patients without atrial fibrillation. ${ }^{10}$ Thus other mechanisms may also be responsible for the higher incidence of thromboembolic events, for example enhanced platelet activation. It has indeed been shown that patients with a ventricular pacemaker have evidence of increased systemic platelet activation. ${ }^{19}{ }^{20}$

PLATELET ACTIVATION AND THROMBOEMBOLISM Alterations in all phases of haemostasis are associated with thromboembolic events; however, platelet activation occurs in the earliest phase of arterial haemostasis and plays an important role in these disorders. Platelet hyperactivity and in vivo platelet activation have been extensively investigated in all major arterial thrombotic disorders, using different methods. ${ }^{21-27}$ These clinical and epidemiological studies provide strong evidence for an association between platelet hyperreactivity and clinical ischaemic disorders. Although a direct causal relation between platelet activation and thromboembolism is lacking, a previous prospective study showed that platelet hyperreactivity predicted an increased risk of future ischaemic events. ${ }^{28} \mathrm{~A}$ recent study also found that plasma $\beta$ thromboglobulin is an independent risk factor for subclinical early carotid atherosclerosis. ${ }^{29}$ Furthermore, the fact that antiplatelet agents are effective in the secondary prevention of thromboembolism ${ }^{13} 30$ provides indirect evidence for this association.

\section{PLATELET ACTIVATION DURING PACING}

It is unclear whether increased platelet activation occurs in patients implanted with pacemakers, or what the underlying mechanism might be. This is the first study to compare platelet activation in different modes of pacing and to provide insight into the possible mechanism of increased platelet activation in ventricular pacing. Our study shows that all patients implanted with a pacemaker have a significant increase in plasma $\mathrm{PF} 4$ and $\beta$ thromboglobulin compared with controls. PF4 and $\beta$ thromboglobulin are specific platelet proteins which are stored in the $\alpha$ granules and 
are released into the plasma during platelet activation. Both are secreted in similar amount in vitro, although the plasma concentration of $\beta$ thromboglobulin is almost always higher than that of PF4, because of difference in clearance (see Methods). Plasma PF4 and $\beta$ thromboglobulin were both significantly raised in paced patients during different pacing modes in comparison with controls. The rise in $\beta$ thromboglobulin was greater than the rise in $\mathrm{PF} 4$, the ratio of $\beta$ thromboglobulin to $\mathrm{PF} 4$ being more than 3 , which is compatible with in vivo activation. ${ }^{16}{ }^{17}$ This suggested that either the presence of the pacemaker and leads or the underlying disease state leads to increased platelet activation. None of the paced patients had a history of diseases that are associated with platelet activation. Furthermore, there was no significant difference in the degree of platelet activation between patients with complete heart block and those with sick sinus syndrome in each respective pacing mode, indicating that the disease status did not affect platelet activation. Thus the enhanced platelet activation is likely to be relate to contact of platelets with an artificial surface.

POSSIBLE MECHANISMS OF PLATELET ACTIVATION ASSOCIATED WITH VENTRICULAR PACING

With the patients acting as their own controls, we compared platelet activation during different modes of pacing. VVIR pacing was associated with significantly higher plasma concentrations of PF4 and $\beta$ thromboglobulin than DDD and DDDR pacing. This suggested that the single ventricular pacing mode led to a higher degree of platelet activation than dual chamber synchronous atrioventricular pacing modes. The possible mechanisms of increased platelet activation during ventricular pacing include (1) haemodynamic irregularities with abnormal blood flow due to atrioventricular asynchrony; (2) the presence of coexisting atrial tachyarrhythmias; (3) differences in the percentage of ventricular pacing; and (4) differences in heart rate attained.

The incidence of atrial tachyarrhythmias was low and short lasting in all three modes of pacing in the study period. Thus the recorded episodes of paroxysmal atrial fibrillation could not account for the difference in plasma $\beta$ thromboglobulin and PF4, which reflect continuous platelet activation in vivo. In addition, it is unclear whether paroxysmal atrial fibrillation could lead to platelet activation as in the case of chronic atrial fibrillation. ${ }^{31}$ Finally, there was no relation between platelet activation and the frequency of ventricular pacing, suggesting that ventricular pacing per se did not increase platelet activation. In fact, as shown in our study and in a previous one, ${ }^{32}$ the percentage of ventricular pacing is actually increased in dual chamber compared with single chamber pacing in patients with the sick sinus syndrome. This is because synchronous ventricular pacing occurs with every $P$ wave in the dual mode. Furthermore, no significant difference in mean heart rate between the different pacing modes was noted to account for the difference in platelet activation. There was also no difference in platelet activation between DDD and DDDR modes, so a loss of rate response was not associated with increased platelet activation. Thus our results suggest that loss of atrioventricular synchrony related to single chamber ventricular pacing is a major cause of increased platelet activation. Spontaneous platelet activation may be related to mechanical force and shear stress elicited by the haemodynamic irregularities caused by atrioventricular asynchrony. This asynchrony is reduced by dual chamber pacing.

\section{CLINICAL IMPLICATION}

Single chamber ventricular pacing with atrioventricular asynchrony was associated with enhanced spontaneous systemic platelet activation. This may be one of the mechanisms for the higher incidence of thromboembolism associated with ventricular pacing, which can be prevented by a physiological pacing mode that maintain atrioventricular synchrony. The increased platelet activation was not related to the presence of the pacing system and the underlying arrhythmias per se, and was independent of the frequency of ventricular pacing, mean heart rate, and rate adaptation. In patients implanted with a ventricular pacemaker who are susceptible to thromboembolism, treatment with antiplatelet agents should be considered on pathophysiological grounds.

The assistance of Dr M O Tang, research assistant in the department, is gratefully acknowledged. This study was supported by the RGC grant (HKU 37/91, grant code 338/041/ 0004). Professor C-P Lau and Dr H-F Tse are members of the Institute of Cardiovascular Science and Medicine.

1 Furman S, Robinson G. The use of an intracardiac pacemaker in the correction of total heart block. Surg Forum 1958;9:245.

2 Kaul T, Kumar EB, Thomson RM, Bain WH. Sinoatrial disorder; "the sick sinus" syndrome. Experience with implanted cardiac pacemaker. F Cardiovasc Surg 1978;19: 261-6.

3 Breivik K, Ohm OJ, Segadal L. Sick sinus syndrome treated with permanent pacemaker in 109 patients. Acta Med with permanent pacenal

4 Stone JM, Bhakta RD, Lutgen J. Dual chamber sequential pacing management of sinus node dysfunction: advantage over single chamber pacing. Am Heart $\mathcal{f}$ 1982;104:131927.

5 Sutton R, Kenny RA. The natural history of sick sinus syndrome. Pacing Clin Electrophysiol 1986;9:1110-14.

6 Rosenqvist M, Brandt J, Schuller H. Long-term pacing in sinus node disease: effects of stimulation mode on cardiovascular morbidity and mortality. Am Heart $\mathcal{7}$ 1988;116:16-22.

7 Langenfeld H, Grimm W, Maisch B, Kochsiek K. Atrial fibrillation and embolic complications in paced patients. Pacing Clin Electrophysiol 1988;11:1667-72.

8 Hesselson AB, Parsonnet V, Bernstein AD, Bonavita GJ. Deleterious effects of long term single chamber ventricular pacing in patients with sick sinus syndrome: the hidden pacing in patients with sick sinus syndrome: the hidden 1992;19:1542-9.

9 Sgarboss EB, Pinski SL, Maloney JD, Simmons TW, Wilkoff BL, Castle LW, et al. Chronic atrial fibrillation and stroke in paced patients with sick sinus syndrome: relevance of clinical characteristics and pacing modalities. Circulation 1993;88:1045-53.

10 Andersen HR, Thuesen L, Bagger JP, Vesterlund T, Thomsen PEB. Prospective randomised trial of atrial versus ventricular pacing in sick-sinus syndrome. Lancet 1994;344: 1523-8.

11 Levine PH, Fisher M, Fullerton AL, Duffy CP, Hoogasian JJ. Human platelet factor IV: preparation from outdated platelet concentrates and application in cerebral vascular disease. Am f Hematol 1981;10:375-85.

12 Stewart ME, Douglas JT, Lowe GD, Prentice CR, Forbes CD. Prognostic value of beta-thromboglobulin in patients with transient cerebral ischemia. Lancet 1983;ii:479-82.

13 Patrono C. Aspirin as an anti-platelet drug. N Engl $7 \mathrm{Med}$ 1994;330:1287-94.

14 Files J, Malpass T, Yee E, Ritchie J, Harker L. Studies of human platelet alpha granule release in vivo. Blood 1981;58:607-18. 
$15 \mathrm{Wu}$ KK. Platelet activation mechanisms and markers in arterial thrombosis. F Intern Med 1996;239:17-34.

16 Kaplan KL, Owen J. Plasma levels of $\beta$-thromboglobulin and platelet factors 4 as indices of platelet activation in vivo. Blood 1981:57:199-202.

17 Kaplan KL. Laboratory marker of platelet activation. In Colman RW, Hirsh J, Marder VJ, Salzman EW, eds. Hemos tasis and thrombosis: basic principles and clinical practice, $3 \mathrm{rd}$ ed. Philadelphia: Lippincott, 1994:1180-1.

18 Sutton R, Markowitz T. Toward physiological stimulation in sinus node disease. Eur 7 Cardiac Pacing Electrophysiol 1994;2:126-31.

19 Fazio S, Cittadini A, Sabatini D, Santomauro M, Cocozza $\mathrm{M}$, Oliviero $\mathrm{U}$, et al. Platelet aggregability in patients with a VVI pacemaker. Pacing Clin Electrophysiol 1993;16:254-6.

20 Fazio S, Santomauro M, Cittadini A, Ferraro S, Lucariello A, Maddalena G, et al. Efficacy of ticlopidine in the prevention of thromboembolic events in patients with VVI

pacemaker. Pacing Clin Electrophysiol 1991;14:168-73.

$21 \mathrm{Wu} \mathrm{KK}$, Hoak JC. A new method for quantitative detection of platelet aggregates in patients with arterial insufficiency. Lancet 1974; ii:924-6.

22 Mehta P, Mehta J. Platelet function studies in coronary artery disease. V. Evidence of enhanced platelet micro thrombus formation activity in acute myocardial infarction Am f Cardiol 1977; 43:757-60.

23 Dougherty JH, Levy DE, Weeksler BB. Platelet activation in acute cerebral ischaemia. Lancet $1977 ; \mathrm{i}: 821-4$
24 Shah AB, Beamer N, Coull BM. Enhanced in-vivo platelet activation in subtypes of ischemic stroke. Stroke 1985;16: activation $643-7$.

25 Sally N, Dugdale $M$. Platelet abnormalities in ischemic heart disease. Am f Cardiol 1973;32:612-8.

26 Rasi V, Ikkala E, Torstila I. Plasma $\beta$-thromboglobulin in acute myocardial infarction. Thromb Res 1982;25:203-12.

27 FitzGerald DJ, Rey L, Catella F, FitzGerald GA. Platelet activation in unstable coronary disease. $N$ Engl $\mathcal{f ~ M e d ~}$ 1986;313:1194-210

28 Trip MD, Cats VM, van Capelle FJ, Vreeken J. Platelet hyperreactivity and prognosis in survivors of myocardial infarction. N Engl f Med 1990;323:1549-54.

29 Ghaddar HB, Cortes J, Saloma V, Kark JD, Davis CE, Folson $\mathrm{AR}$, et al. Correlation of specific platelet activation markers with carotid artery wall thickness. Thromb Haemost 1995;74:943-8.

30 Antiplatelet Trialists' Collaboration. Secondary prevention of vascular disease by prolonged antiplatelet therapy. $B M \mathcal{F}$ 1988;296:320-31.

31 Yamauchi K, Furui H, Taniguchi N, Sotobata I. Plasma $\beta$-thromboglobulin and platelet factor 4 concentration in patients with atrial fibrillation. fpn Heart f 1986;27:481-7.

Lau CP, Tai YT, Leung WH, Wong CK, Lee P, Chung LW. Rate adaptive pacing in sick sinus syndrome: effects of pacing modes and intrinsic conduction on physiological responses, arrhythmias, symptomatology and quality of life. Eur Heart 7 1994;15:1445-55. 